\title{
Isolated Axillary Nerve Injury following a Blunt Trauma
}

\author{
Muhammad Fawwaz Ramli${ }^{1}$, Muhammad Wafiuddin², Mahyuddin Mohamed ${ }^{1}$ \\ ${ }^{1}$ Department of Orthopeadic, Hospital Sultan Haji Ahmad Shah, Temerloh Pahang \\ ${ }^{2}$ Department of Surgical-Based, Faculty of Medicine and Health Sciences, Universiti \\ Malaysia Sabah.
}

Introduction: Axillary nerve supplies deltoid muscle long head of triceps and teres minor in the arm and supply regimental batch area over the inferior part of shoulder. Anterior dislocation of shoulder, latrogenic injury to the nerve during shouder injection and brachial plexus injury can cause axillary nerve injury. Case report: We report a rare case of isolated axillary nerve injury in a 20 year old lady. She was admitted following a low speed fall from motorcycle and sustain abrasion wound over the right side of the face and shoulder. There was no direct or tractional injury to the shoulder during the incidence.During admission she complaint of progressive right shoulder weakness with MRC power of 2 and sensory loss over regimental patch area. On examination there was no tenderness to the shoulder area or neck and she was unable to abduct her right shoulder with full passive range of motion. Patient was subjected to nerve conduction study four months later and revealed slower sensory conduction and deltoid muscle amplitude decreased compared the left side. At 7 months post trauma she is recovering with deltoid power of 4 after multiple physiotherapy session. Isolated axillary nerve injury following a blunt trauma is a rare condition with multiple reported cases showed poor recovery. One should wait for regeneration of nerves to complete before offering any surgical intervention to the patient. As in our case, patient showed remarkable improvement after seven months with conservative management thus avoiding the complication of surgery. 